\title{
SURFACE LATTICE RESONANCES IN PLASMONIC ARRAYS OF ASYMMETRIC DISC DIMERS
}

\section{- SUPPORTING INFORMATION -}

\author{
Alastair D. Humphrey, Nina Meinzer*, Timothy A. Starkey, and William L. Barnes \\ School of Physics and Astronomy \\ University of Exeter \\ Stocker Road \\ Exeter \\ EX4 4QL \\ United Kingdom
}

\section{MODIFIED S-FACTOR MODEL}

The $S$-factor ${ }^{1,2,3,4}$ describes the electric field contribution $E_{A}$ of the array surrounding a particle to the total electric field at the position of the particle, taking into account the sum of the scattered electric fields from other particles in the arrangement, which add to the incident electric field $E_{0}$.

$$
E_{\mathrm{tot}}=E_{0}+E_{A}=E_{0}+S P \text {, }
$$

where $P$ is the effective total polarizability of the array

$$
P=\alpha^{*} \varepsilon_{0} E_{0}=\left(\frac{\alpha}{1-\alpha \varepsilon_{0} S}\right) \varepsilon_{0} E_{0},
$$

defining the effective particle polarizability $\alpha^{*}$.

In order to find the expression for the effective polarizability in an array with a base element made up of two (different) particles, as given in equation 1 of the main paper, we need to consider the total electric fields at the position of particles 1 and 2 separately (for a more detailed treatment see reference 5). Each particle (labelled as 1 and 2) is described with a corresponding polarizability

$$
P_{1,2}=\alpha_{1,2} \varepsilon_{0} E_{1,2}=\alpha_{1,2}^{*} \varepsilon_{0} E_{0},
$$

with $E_{1,2}$ the total electric fields at the position of particle 1 and 2, respectively. Equation $S 1$ is then replaced by two equations taking into account the scattered fields from the same type as the reference particle - described by $S$ - and from the other type of particle - described by $S^{\prime}$.

$$
\begin{aligned}
& E_{1}=E_{0}+S P_{1}+S^{\prime} P_{2}, \\
& E_{2}=E_{0}+S^{\prime} P_{1}+S P_{2} .
\end{aligned}
$$

Combining equations S3, S4 and S5 and solving for $P_{1}$ leads to

$$
P_{1}=\frac{\alpha_{1}\left(1-\alpha_{2} \varepsilon_{0} S+\alpha_{2} \varepsilon_{0} S^{\prime}\right)}{\left(1-\alpha_{1} \varepsilon_{0} S\right)\left(1-\alpha_{2} \varepsilon_{0} S\right)-\alpha_{1} \alpha_{2}\left(\varepsilon_{0} S^{\prime}\right)^{2}} \varepsilon_{0} E_{0} .
$$


By comparing equation S6 with equation S2, we find an expression for the polarizability of particle 1, modified by all the other particles (both type 1 and 2 ) in the array,

$$
\alpha_{1}^{*}=\frac{\alpha_{1}\left(1-\alpha_{2} \varepsilon_{0} S+\alpha_{2} \varepsilon_{0} S^{\prime}\right)}{\left(1-\alpha_{1} \varepsilon_{0} S\right)\left(1-\alpha_{2} \varepsilon_{0} S\right)-\alpha_{1} \alpha_{2}\left(\varepsilon_{0} S^{\prime}\right)^{2}},
$$

or, by an analogous derivation, the modified polarizability for particle 2 ,

$$
\alpha_{2}^{*}=\frac{\alpha_{2}\left(1-\alpha_{1} \varepsilon_{0} S+\alpha_{1} \varepsilon_{0} S^{\prime}\right)}{\left(1-\alpha_{1} \varepsilon_{0} S\right)\left(1-\alpha_{2} \varepsilon_{0} S\right)-\alpha_{1} \alpha_{2}\left(\varepsilon_{0} S^{\prime}\right)^{2}} .
$$

\section{INFLUENCE OF OFF-AXIS ELEMENTS}

In order to obtain a better understanding of how the interaction of all elements in a square array of asymmetric disc dimers (ADDs) gives rise to the spectra observed, we distinguish between contributions from elements along the two array axes ( $x$ and $y$ ) and those from offaxis elements, which are out of phase with the fields scattered from on-axis elements. Using S-factor modeling, we calculated extinction cross-section spectra for one-dimensional ADD chains with elements repeated along the $x$ and $y$ axis, respectively, as well as for a square array (ADD dimensions are $d_{1}=85 \mathrm{~nm}, d_{2}=115 \mathrm{~nm}$, center-to-center separation $c=150 \mathrm{~nm}$ and the lattice constant is $a=450 \mathrm{~nm}$ in all cases). The results are shown in figure $\mathrm{S} 1 \mathrm{for}$ incident polarization (a) parallel to and (b) perpendicular to the dimer axis.

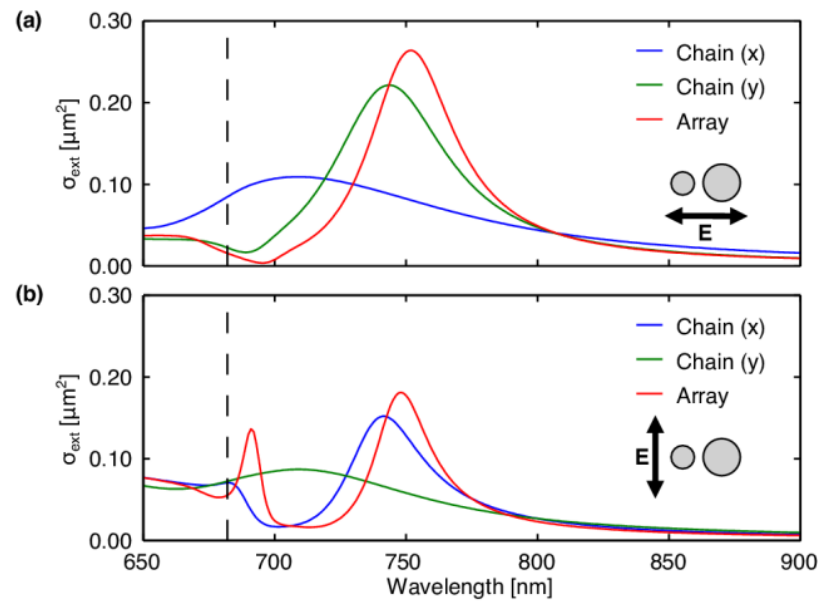

Figure S1. Comparison of $S$-factor models for one- and two-dimensional ADD arrangements. Extinction cross sections per dimer $\left(d_{1}=85 \mathrm{~nm}, d_{2}=115 \mathrm{~nm}, c=150\right.$ $\mathrm{nm})$ in one-dimensional chains $(a=450 \mathrm{~nm})$ arranged along the $x$ axis and $y$ axis, and in square lattices $(a=450 \mathrm{~nm})$ for an incident electric field oriented (a) parallel and (b) perpendicular to the dimer axis.

Comparing the spectra for the square array (red) with those of the chains along the $x$ axis (blue) and the $y$ axis (green) we can see that, for wavelengths significantly longer than the diffraction edge (dashed black line), the array response is given by the sum of the two chain contributions, indicating that the contribution of off-axis elements is negligible. However, this is not the case for wavelengths around the diffraction edge, where we find a marked difference between the response of the array and that of the chains, which is most evident from Fig. S1a: For polarization parallel to the dimer axis the extinction cross-section of the array is almost 
zero although both directions of ADD chains have non-zero extinction cross-sections of greater values than the array. This can only be explained by taking into account the destructive interference with the (out-of-phase) scattered electric fields originating from off-axis elements.

\section{REFERENCES}

(1) Markel V. A. Coupled-dipole approach to scattering of light from a one-dimensional periodic dipole structure. J. Mod. Opt. 1993, 40, 2281-2291.

(2) Zhou, S.; Janel; N. Schatz, G. C.; Silver nanoparticle array structures that produce remarkably narrow plasmon lineshapes. J. Chem. Phys. 2004, 120, 10871.

(3) Markel. V. A. Divergence of dipole sums and the nature of non-Lorentzian exponentially narrow resonances in one-dimensional periodic arrays of nanospheres. J. Phys. B: At. Mol. Opt. Phys. 2005, 38, L115.

(4) García de Abajo, F. J. Colloquium: Light scattering by particle and hole arrays. Rev. Mod. Phys. 2007, 79, 1267.

(5) Humphrey, A. D.; Barnes, W. L. Plasmonic surface lattice resonances in arrays of metallic nanoparticle dimers. J. Opt. 2016, 18, 035005. 